\title{
Inhibition of Notch Signaling Promotes the Adipogenic Differentiation of Mesenchymal Stem Cells Through Autophagy Activation and PTEN-PI3K/ AKT/mTOR Pathway
}

\author{
Bao-quan Song Ying Chi Xue Li Wen-jing Du Zhi-Bo Han Jian-jian Tian \\ Juan-juan Li Fang Chen He-he Wu Li-xin Han Shi-hong Lu Yi-zhou Zheng \\ Zhong-chao Han
}

State Key Laboratory of Experimental Hematology, National Engineering Research Center of Stem Cells, Institute of Hematology and Hospital of Blood Diseases, Chinese Academy of Medical Sciences \& Peking Union Medical College, Tianjin, China

\section{Key Words}

Notch signaling • Mesenchymal stem cells • Adipogenesis • Autophagy • Phosphoinositide 3-kinase $\cdot$ Akt $\cdot \mathrm{mTOR}$

\begin{abstract}
Background: The Notch signaling pathway is implicated in a broad range of developmental processes, including cell fate decisions. This study was designed to determine the role of Notch signaling in adipogenic differentiation of human bone marrow derived MSCs (BMMSCs). Methods: The Notch signaling was inhibited by the $\gamma$-secretase inhibitor $\mathrm{N}-[\mathrm{N}-(3,5-$ difluor- ophenacetyl-L-alanyl)]-S-phenylglycine t-butylester (DAPT). The markers involving adipogenic differentiation of MSCs, the relative pathway PTEN-PI3K/Akt/mTOR and autophagy activation were then analyzed. Furthermore, the autophagy inhibitor chloroquine (CQ) and 3-methyladenine (3-MA) were used to study the role of autophagy in the DAPTinduced the adipogenic differentiation of MSCs. Results: We first confirmed the downregulation of Notch gene expression during MSCs adipocyte differentiation, and showed that the inhibition of Notch signaling significantly enhanced adipogenic differentiation of MSCs. Furthermore, Notch inhibitor DAPT induced early autophagy by acting on PTEN-PI3K/Akt/ mTOR pathway. The autophagy inhibitor CQ and 3-MA dramatically abolished the effects of DAPT-induced autophagy and adipogenic differentiation of MSCs. Conclusion: Our results indicate that inhibition of Notch signaling could promote MSCs adipogenesis mediated by autophagy involving PTEN-PI3K/Akt/mTOR pathway. Notch signaling could be a novel target for regulating the adipogenic differentiation of MSCs.
\end{abstract}




\section{Cellular Physiology Cell Physiol Biochem 2015;36:1991-2002 \begin{tabular}{l|l} 
and Biochemistry Published onIIne: July 17, 2015 & $\begin{array}{l}\text { C) 2015 S. Karger AG, Basel } \\
\text { www.karger.com/cpb }\end{array}$ \\
\hline
\end{tabular} \\ Song et al.: Notch Signaling Regulates Adipogenesis via Autophagy and PTEN-PI3K/ AKT/mTOR Pathway}

\section{Introduction}

Human adult mesenchymal stem cells (MSCs) are a population of stromal cells present in bone marrow and many other tissues, which can differentiate into various cell types including osteoblasts, chondrocytes and adipocytes [1-3]. During the process of aging or in some pathological conditions, including aplastic anemia, multiple myeloma, and anorexia nervosa, the cell lineage commitment of MSCs shift to adipocytes, especially in bone marrow [4-7]. Over adipogenesis will usually lead to obesity, a major risk factor for certain chronic diseases, such as cardiovascular disease and type 2 diabetes [8, 9]. As adipocytes can be traced back to a common precursor MSCs, the precise molecular mechanism governing the adipogenic differentiation of MSCs becomes an important issue in this field.

Previous studies have shown that the adipogenic differentiation of MSCs is a complicated physiological process that involves a large number of molecular events. Various reports have demonstrated that under defined conditions in vitro, MSCs can differentiate into adipocytes depending on the presence of some particular cytokines (e.g, IFN- $\gamma$, TNF- $\alpha$, TGF- $\beta$, and BMPs) and the extracellular matrix $[10,11]$. It's also documented that the microRNAs (miRNAs) exert essential regulatory functions in adipocyte development [12]. However, the cellintrinsic mechanism of this cell lineage commitment switch is poorly understood. Autophagy is a multifaceted process to degrade intracellular components by forming autophagosomes, which then fuse with lysosomes to form autolysosomes $[13,14]$. Growing evidence suggests that autophagy is implicated in adipogenesis [15] and adipogenic differentiation [16].

Notch signaling is a developmental pathway that regulates several fundamental cellular processes including cell fate and differentiation [17, 18]. Four transmembrane Notch receptors (Notch-1, -2, -3, -4), the five canonical ligands (DLL1, DLL3, DLL4, JAGGED1, and JAGGED2), and the two non-canonical ligands (DLK1 and DLK2) constitute the Notch system [19]. Notch activation occurs when the ligand engagement renders the Notch intracellular domain to be cleaved from the cell membrane and translocate into the nucleus, where it forms a transcriptional activator complex with the CSL family of transcription factors (C-promoter binding factor $1 /$ recombination signal sequence binding protein $\mathrm{J} \kappa$, suppressor of hairless, and Lag-1) and modulates the expressions of target genes such as Hes family of transcription factors members and p21 $[20,21]$. The final cleavage that untethered Notch receptors from the cell membrane is mediated by the enzymatic action of $\gamma$-secretase and can be blocked with pharmacological inhibitors. Follow-up studies demonstrated that $\gamma$-Secretase inhibitors (DAPT) successfully prevent the final enzymatic step required for Notch cleavage and activation and can block Notch signaling in vitro and in vivo [18, 22]. Moreover, recent evidence has found that the components of the Notch pathway are expressed and activated in T-cell development, megakaryocyte development, marginal zone B cells development and HSC differentiation [23-26]. However, little is known about the effect of the Notch signaling pathway on human mesenchymal stem cell differentiation.

The present study was designed to determine the role of Notch signaling involved in human bone marrow derived MSCs (BM-MSCs) in terms of adipogenic differentiation and further disclose the potential molecular mechanism. The data reveal that inhibition of Notch signaling promotes BM-MSCs adipogenesis mediated by autophagy activation involving PTEN-PI3K/Akt/mTOR pathway.

\section{Materials and Methods}

\section{Reagents and antibodies}

3-methyladenine (3-MA), chloroquine (CQ) and DAPT were purchased from Sigma (St. Louis, MO, USA). The following antibodies were from Cell Signaling Technology (Beverly, MA, USA): anti- $\beta$-actin, anti-LC3II/I, anti-mTOR, anti-phosphorylation of mTOR (p-mTOR), anti-Akt, anti-phosphorylation of Akt (p-Akt). The anti-bodies of anti-PI3K and anti-phosphorylation of anti-PI3K were from Santa Cruz Biotechnology (Santa Cruz, CA, USA), and anti-beclin1 was obtained from Pepro Tech (Proteintech, USA). 
Cell culture and differentiation

This study was approved by the Institutional Review Board of Chinese Academy of Medical Sciences and Peking Union Medical College. Bone marrow samples were obtained from donors with written informed consent. The isolation and expansion of BM-MSCs were performed as described previously described [4]. BM-MSCs were maintained in DMEM/F-12 medium (Gibco) containing 10\% fetal bovine serum (FBS, HyClone), $2 \mathrm{mM}$ glutamine, $100 \mathrm{U} / \mathrm{ml}$ penicillin-streptomycin, and $10 \mathrm{ng} / \mathrm{ml}$ epidermal growth factor (EGF; Pepro Tech), incubated at $37^{\circ} \mathrm{C}$ and a $5 \% \mathrm{CO}_{2}$ humid atmosphere, cells from passage 3-5 were used for the following experiments. DAPT was dissolved in DMSO, and was freshly diluted to the desired concentration with culture medium. The final concentration of DMSO was at $0.05 \%(\mathrm{v} / \mathrm{v})$. The DMSO group cells received the vehicle only.

For osteogenic induction, basal medium was replaced with differentiation medium consisting of IMDM supplemented with $0.1 \mu \mathrm{M}$ dexamethasone, $200 \mu \mathrm{M}$ ascorbic acid-2-phosphate, $10 \mathrm{mM} \beta$-glycerophosphate, and $10 \%$ FBS. For adipogenic induction, medium consisted of IMDM supplemented with $1 \mu \mathrm{M}$ dexamethasone,

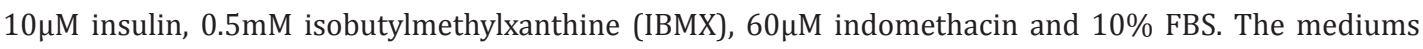
were replaced three times a week.

\section{Oil Red 0 staining}

After adipogenic induction, BM-MSCs were stained with Oil red 0. Cells were washed twice with PBS and fixed in $4 \%$ paraformaldehyde at room temperature for $30 \mathrm{~min}$. Then, the cells were stained with an Oil red $\mathrm{O}$ solution $\left(60 \%\right.$ Oil Red $\mathrm{O}$ stock solution and $\left.40 \% \mathrm{H}_{2} \mathrm{O}\right)$ for $15 \mathrm{~min}$ and then washed three times with PBS. The stained lipid drops were observed using an inverted microscope.

\section{Quantitative real-time \\ PCR}

Total RNA was extracted from cells using TRIZOL ${ }^{\circledR}$ Reagent (Invitrogen, Carlsbad, CA) according to the manufacturer's instructions. cDNA was synthesized using EasyScript First-Strand cDNA Synthesis SuperMix (TransGen Biotech China Co.,Ltd. Beijing). Real-time PCR was carried out with an ABI 7500 real-time PCR system using SYBR Green mix (ABI, USA). The fold-change in gene expression relative to the control was calculated by $2^{-(\triangle \Delta C T)}$. The experiments were repeated 3 times and every sample was performed in triplicate. Primers used in the experiment were listed in Table 1.

Table 1. Sequence of primers used in real-time PCR

\begin{tabular}{|c|c|c|}
\hline Genes & Forward primer & Reverse primer \\
\hline NOTCH 1 & 5'-TCAGCGGGATCCACTGTGAG-3' & 5'-ACACAGGCAGGTGAACGACTTG-3' \\
\hline NOTCH 2 & 5'-ACAGTTGTGTCTGCTCACCAGGAT-3' & 5'-GCGGAAACCATTCACACCGTTGAT-3' \\
\hline NOTCH 3 & 5'-GATGGCATGGATGTCAATGTTCGT-3' & 5'-TGCCTCATCCTCTTCAGTTGGCAT-3' \\
\hline NOTCH 4 & 5'-TCAAACAGAGGTGGATGAGTGCCT-3' & 5'-AGTTGGCCTTGTCTTTCTGGTCCT-3' \\
\hline JAGGED1 & 5'-ACTGCTCACACCTGAAAGACCACT-3 & 5'-AGGACCACAGACGTTGGAGGAAAT-3' \\
\hline JAGGED2 & 5'-TGCTGTGGAGGTGGCTATGTCT-3' & 5'-TGTTTCCACCTTGACCTCGGT-3' \\
\hline DLL1 & 5'-TGTGACGAGTGTATCCGCTATCCA-3' & 5'-AGGGCTTATGGTGTGTGCAGTAGT-3' \\
\hline DLL3 & 5'-TCCCGGATGCACTCAACAACCTAA-3' & 5'- TTCAGGGCGATTCCAATCTACGGA-3' \\
\hline DLL4 & 5'-ACTGCGAGAAGAAAGTGGACAGGT-3' & 5'-ACATGAGCCCATTCTCCAGGTCAT-3' \\
\hline DLK1 & 5'-TGGACGATGGCCTCTATGAATGCT-3' & 5'-TCTCGCAGAAATTGCCTGAGAAGC-3' \\
\hline DLK2 & 5'-CCATTGTGTGTGCTTACCAGGCTT-3' & 5'-CGCATCAGGCAGTCATCCACATTT-3' \\
\hline HES1 & 5'-GTCAACACGACACCGGATAAACCA-3' & 5'-TTTCCAGAATGTCCGCCTTCTCCA-3' \\
\hline HEY1 & 5'-AGAGTGCGGACGAGAATGGAAACT- 3' & 5'-ACCAGCCTTCTCAGCTCAGACAAA-3' \\
\hline HEY2 & 5'-AGATGCTTCAGGCAACAGGG-3' & 5'-CAAGAGCGTGTGCGTCAAAG-3' \\
\hline PPAR $\gamma$ & 5'-AGCCTCATGAAGAGCCTTCCA-3' & 5'-ACCCTTGCATCCTTCACAAGC-3' \\
\hline $\mathrm{C} / \mathrm{EBP} \alpha$ & 5'-GAAGTTGGTGGAGCTGTCGG-3' & 5'-TGAGGTATGGGTCGTTGCTGA-3' \\
\hline GAPDH & 5'- CGGATTTGGTCGTATTGGGC -3' & 5'- CTTCCCGTTCTCAGCCTTG -3' \\
\hline
\end{tabular}




\section{Cellular Physiology Cell Physiol Biochem 2015;36:1991-2002 \begin{tabular}{l|l} 
and Biochemistry Published onIIne: July 17, 2015 & $\begin{array}{l}\text { C 2015 S. Karger AG, Basel } \\
\text { www.karger.com/cpb }\end{array}$ \\
\hline
\end{tabular} \\ Song et al.: Notch Signaling Regulates Adipogenesis via Autophagy and PTEN-PI3K/ AKT/mTOR Pathway}

\section{Western blot analysis}

BM-MSCs were collected, washed, and lysed with RIPA lysis buffer (Beyotime Institute of Biotechnology, China) supplemented with PMSF (Invitrogen). Total protein was extracted and the protein concentration of the lysates was quantified by the BCA protein assay kit (Pierce). Protein ( $30 \mu \mathrm{g})$ was denatured, separated by SDS-PAGE electrophoresis and transferred to a PVDF membrane. The transferred membranes were blocked using 5\% BSA in TBST and incubated with appropriate primary antibodies overnight and incubated with the corresponding horseradish peroxidase (HRP)-conjugated secondary antibody at 1:2,000 dilution for $2 \mathrm{~h}$. Bands were visualized using enhanced chemiluminescence (ECL, Thermo Scientific, CA) detection reagents and scanned images were quantified using Image J software. The ratio of target gene to $\beta$-actin was used for semi-quantification and comparison among different groups.

\section{Immunofluorescence}

After treated according to protocol, cells were fixed in 4\% paraformaldehyde, permeabilized in $0.25 \%$ Triton, and blocked with Blocking Buffer ( $1 \times \mathrm{PBS}+5 \%$ normal serum $+0.3 \%$ Triton $\left.^{\mathrm{TM} X} \mathrm{X}-100\right)$. Then incubated overnight with rabbit polyclonal anti-LC3B antibody (CST, Beverly, MA, USA), after this incubation, cells were stained for $1 \mathrm{~h}$ with goat anti-rabbit IgG peroxidase antibody (Sigma, St Louis, MO) counterstained with DAPI (Sigma, St Louis, MO), the cells were subsequently viewed by fluorescence microscopy.

\section{Statistical analysis}

The data were presented as mean \pm SD, comparisons of quantitative data were analyzed using Student's t-test between two groups or by one-way ANOVA followed by the Newman-Keuls test for multiple groups. Statistical significance was set at $\mathrm{P}<0.05$. All experiments were performed at least three times.

\section{Results}

\section{Basal characterization of BM-MSCs}

Elongated spindle-shaped or rhomboid BM-MSCs grew colonially and displayed a rather homogeneous confluent population (Fig. 1A). The cells possessed multipotent differentiation potential, as they could be induced into osteoblasts and adipocytes under standard in vitro differential conditions (Fig. 1B and C). Cells showed positive expression of CD44, CD73, CD90, CD105, CD166, but negative for CD14, CD19, CD34, CD45, HLA-DR (Fig. 1D).

\section{Notch Signaling is downregulated during adipocyte differentiation}

To understand the role of Notch signaling during adipocyte differentiation, we investigated the modulation of Notch signaling during adipocyte differentiation through the gene expression of Notch receptors, ligands and the transcription factors by real-time PCR. As show in Fig. 2A, adipogenic differentiation of BM-MSCs was associated with a lower Notch receptors, Notch1, Notch2 and Notch3 than in undifferentiated group. Notch4 expression appeared to be also decrease, although their decrease was not significant. The expression of Notch canonical ligands DLL1, DLL3, DLL4, and the two non-canonical ligands (DLK1and DLK2) were significantly decreased compared to undifferentiated group, whereas there were no significant changes in JAGGED1 and JAGGED2 gene expression (Fig. 2B and C). The gene expressions of the downstream effector genes activated by Notch signaling, HES1, HEY1, and HEY2 were also analyzed. Only HEY1 gene expression was significantly decreased compared to the undifferentiated group, the expression levels of HES1 and HEY2 were also decreased, but they did not reach statistical significance (Fig. 2 D). These findings suggested that Notch signaling could be potential key targets in the adipogenic differentiation of BM-MSCs.

\section{Adipogenic differentiation of BM-MSCs induces early autophagy}

To explore the role of autophagy in adipogenesis, we quantified the levels of autophagy during adipogenic differentiation of BM-MSCs, We found that during initial stages of differentiation, the conversion of LC3-I to autophagosome associated LC3-II, as a marker of autophagy, reaching its maximum at day 3 and then rapidly declined at later stages of 


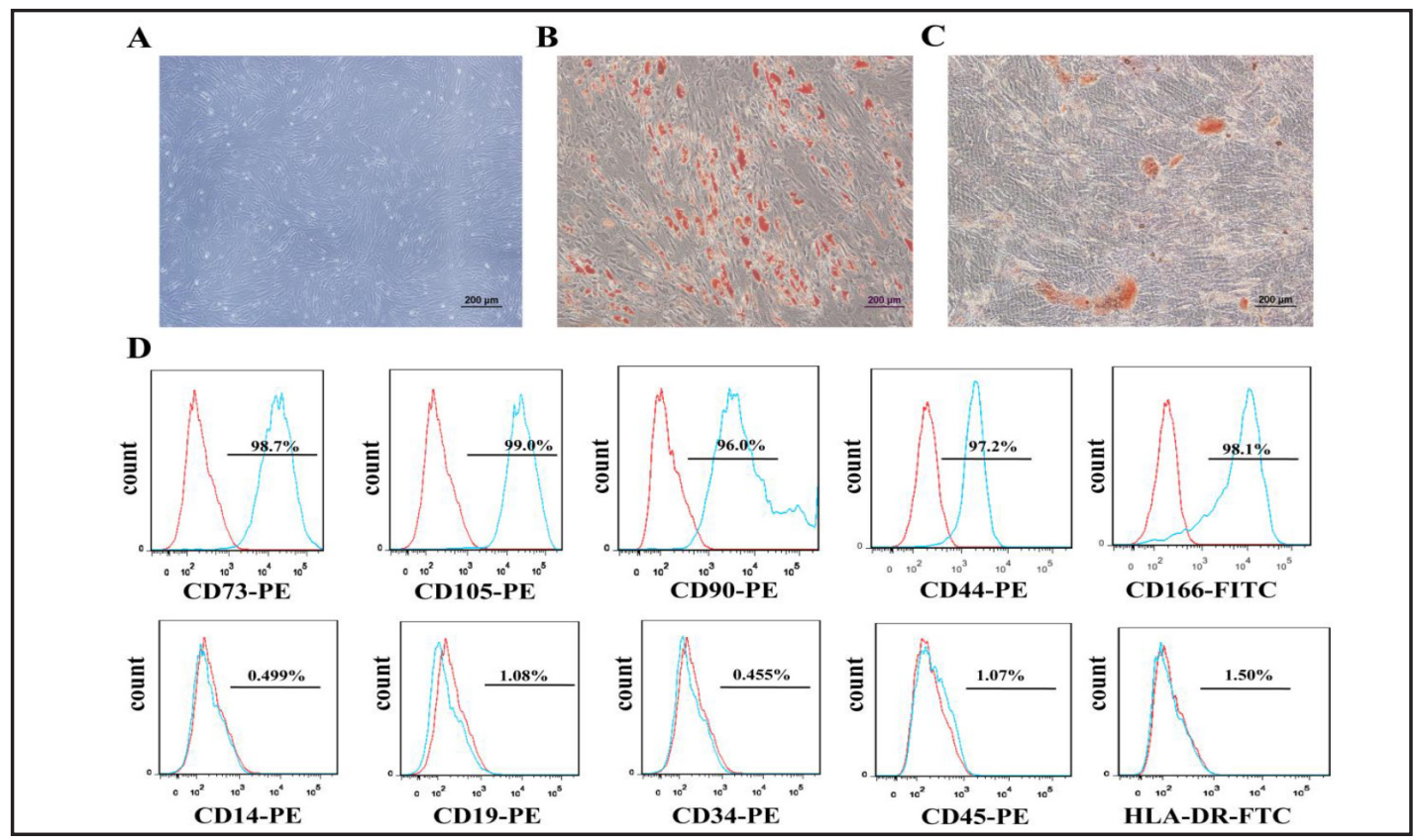

Fig. 1. Characterization of bone marrow derived mesenchymal stem cells (BM-MSC). (A) Morphology of BM-MSCs after culturing the cells for 6 days. (B) Adipocyte induction for 14 days, stained by lipid accumulation with Oil Red O, (C) osteoblast induction for 21 days, mineralized nodules were detected after Alizarin Red S staining. Scale bars $=200 \mu \mathrm{m}$. (D) Immunophenotyping of BM-MSCs by flow cytometry.

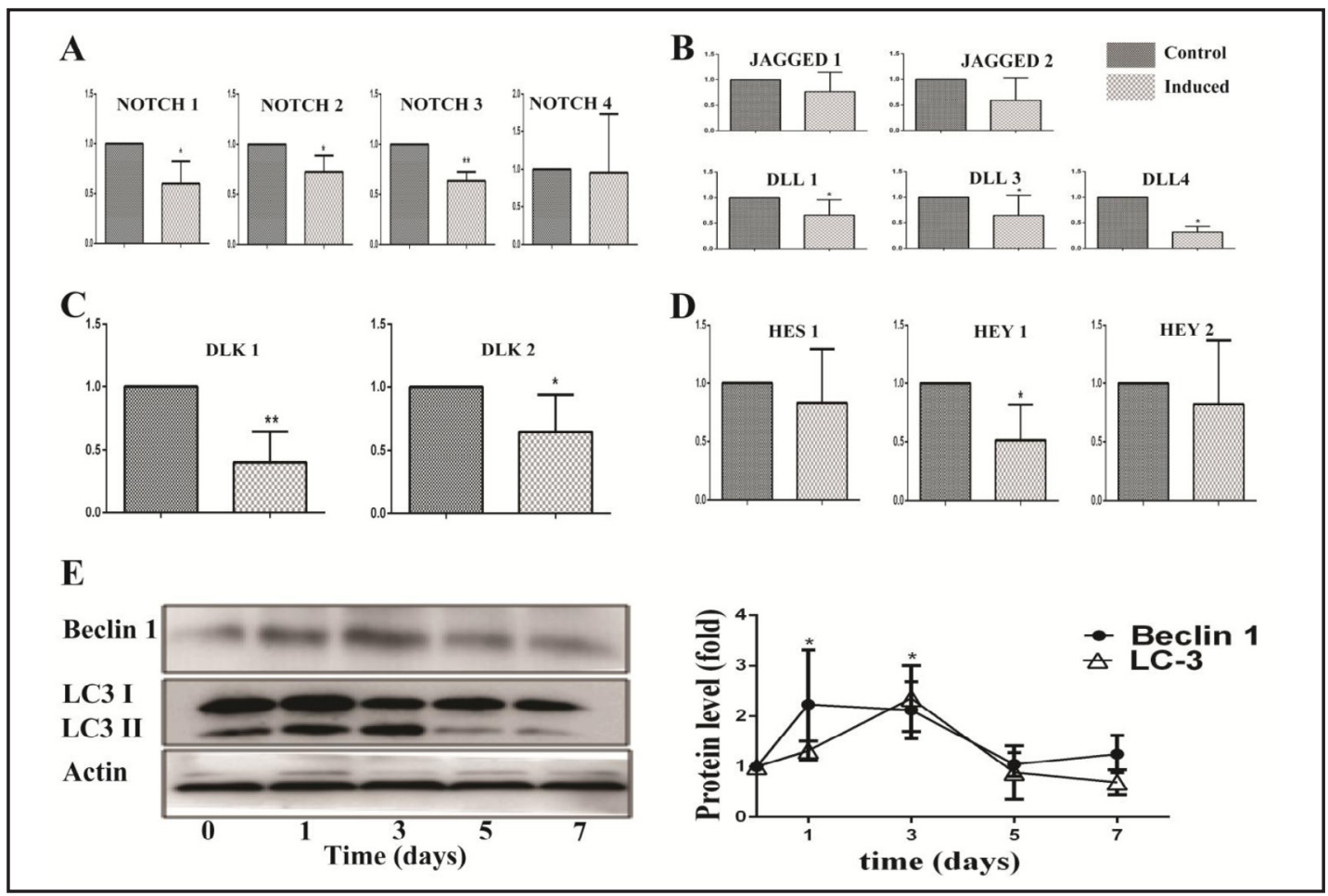

Fig. 2. The changes of Notch signaling and autophagy during adipocyte differentiation. (A) Quantitative real-time PCR was used to determine the four Notch receptor NOTCH1, NOTCH2, NOTCH3 and NOTCH4, (B) Notch canonical ligand genes, Jagged1, Jagged2, DLL1, DLL3, DLL4, (C) Notch non-canonical ligandDLK1 and DLK2. (D). Notch-dependent transcription factors, HES1, HEY2, and HEY2 relative mRNA expression levels, BM-MSCs incubated in differentiation medium for 14 days. (E) Autophagy activation was assessed by western blot at $0,1,3,5$, and $7 \mathrm{~d}$ after adipogenic induction. ${ }^{*} \mathrm{p}<0.05,{ }^{* *} \mathrm{p}<0.01$. 


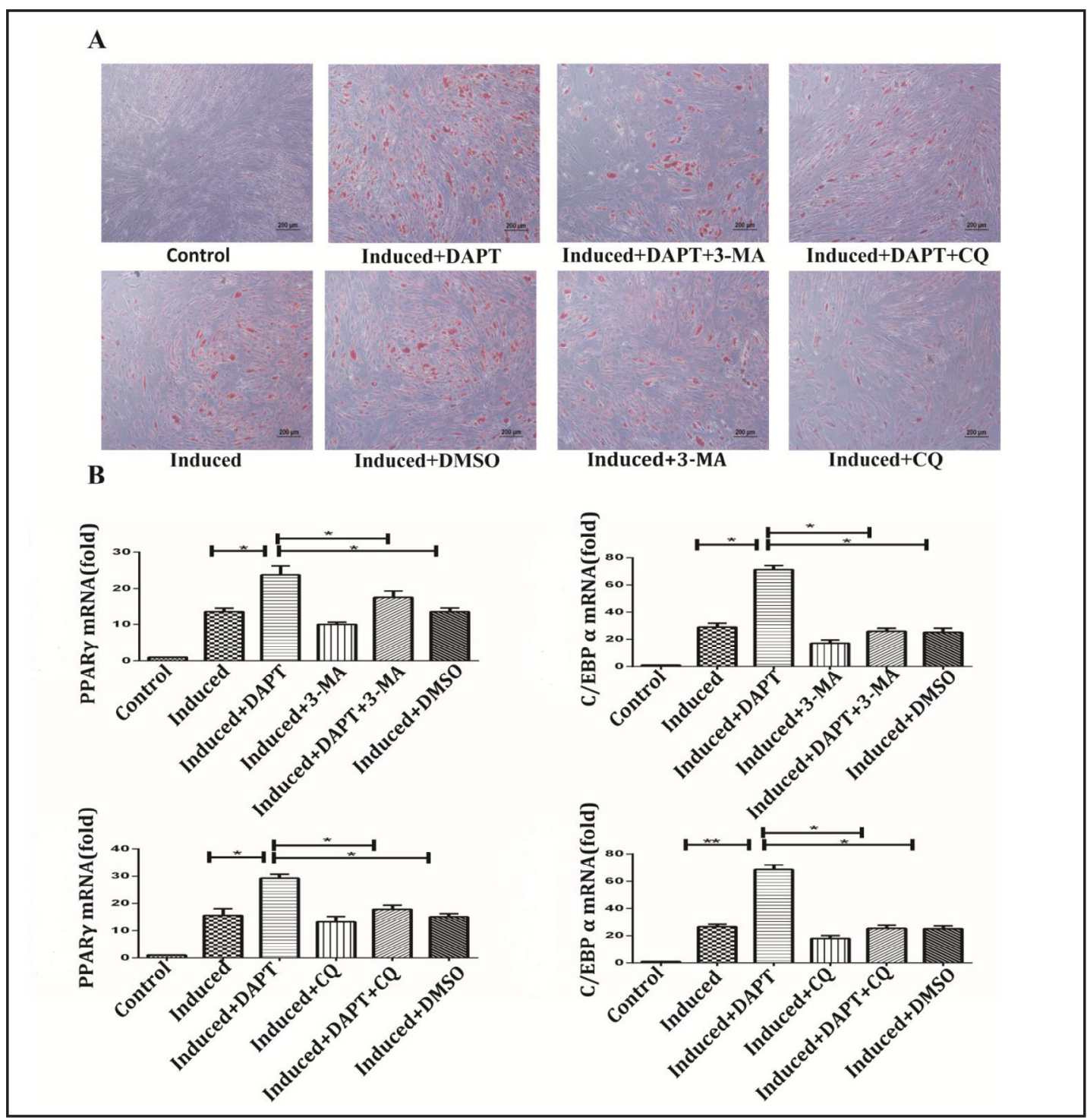

Fig. 3. DAPT promoted adipogenesis by activating autophagy. (A)BM-MSCs were cultured in control mediumor adipogenic medium, in the presence of $5 \mu \mathrm{M}$ DAPT alone, or combine with 3-MA (5mM) or CQ $(20 \mu \mathrm{M})$ for $72 \mathrm{~h}$, after that the cells were subjected to Oil Red 0 staining (Original magnification, 200×); B. mRNA expression of PPAR $\gamma$ and C/EBP $\alpha$ were assessed by real-time PCR. ${ }^{*} \mathrm{p}<0.05$.

differentiation. Changes in the LC3-II/LC3-I ratio were correlated with the extent of proautophagic protein beclin-1, which reached its maximum at day 1 and day 3 after initiation of differentiation (Fig. 2E). The results suggest that autophagy seems a key target in efficient MSC adipogenic differentiation and function.

\section{DAPT promotes the adipogenic differentiation of BM-MSCs through the activation of autophagy}

To evaluate the effects of Notch signaling on the adipogenic differentiation of BM-MSCs, BM-MSCs were allowed to differentiate into adipocytes in the absence or presence of Notch inhibitor DAPT $(5 \mu \mathrm{M})$ during the entire differentiation period 14 days. Following adipocyte induction, significantly increased lipid droplets (Fig. 3A) and mRNA levels of PPAR- $\gamma$ and C/EBP- $\alpha$ (Fig. 3B) were observed in DAPT treated group in comparison with the induced group or DMSO-alone group. These results collectively demonstrate that Notch signaling is

\section{KARGER}




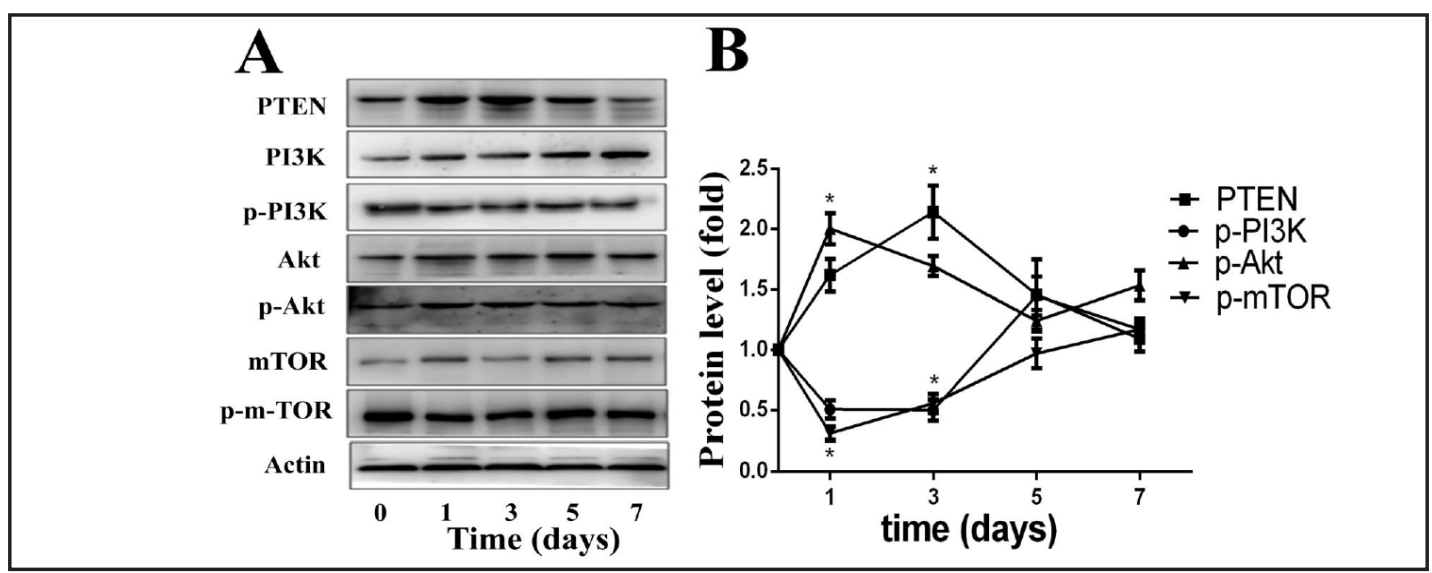

Fig. 4. Modulation of PI3K/Akt/mTOR signaling during adipogenic differentiation of BM-MSCs, the expression of PI3K/Akt/mTOR signaling pathway members was assessed by western blot at the indicated time points. The densitometry data are significantly different in comparison with day $0 .{ }^{*} \mathrm{p}<0.05$.

a critical regulator of adipogenesis and inhibition of Notch signaling promotes adipogenic differentiation of BM-MSCs.

As the activation of autophagy happened after adipogenic induction, particularly during the first three days, we postulated that autophagy might play an important role during adipogenesis stage. To investigate whether the DAPT-induced adipogenesis is dependent on autophagy activation, cells were incubated with autophagy inhibitor 3-MA (5 mM) and CQ $(20 \mu \mathrm{M})$ either alone or in combination with DAPT during adipogenic differentiation conditions for early 3 days. Adipogenesis was assessed by oil $O$ staining and PPAR $\gamma$ and C/EBP $\alpha$ mRNA expression (Fig. 3A, B). We confirmed the addition of 3-MA or CQ to DAPT reversed the pro-adipogenic effect. Taken together, these results show that the effect of DAPT on the adipogenic differentiation of BM-MSCs is partly due to the activation of early autophagy.

Adipogenic differentiation of BM-MSCs is associated with time-dependent modulation of PI3K/Akt/mTOR signaling

To clarify the further molecular mechanism of Notch signaling and autophagy during adipogenic differentiation of BM-MSCs, we examined the expression profiles of PI3K, PTEN, Akt and mTOR at 0, 1, 3, 5, and 7 days after inducing adipogenesis. As shown in Fig. 4A, B, an inverse activation pattern was observed with p-PI3K, demonstrating an early decrease at day 1 followed by activation from day 3 onwards. This process was associated with PTEN increase and rapid phosphorylation of Akt, which reached its peak at day 3 and day 1 respectively, and then gradually declined. The decrease in p-mTOR has roughly paralleled that of p-PI3K, reaching its maximum at day 1 and remaining high during the rest of the differentiation period. These data demonstrated a complex, time-dependent modulation of PI3K/Akt/mTOR signaling during adipogenic differentiation of BM-MSCs.

\section{DAPT induces autophagy in BM-MSCs via inhibition of PI3K/Akt/mTOR pathway}

In order to check whether DAPT could induce autophagy of BM-MSCs and elaborate the underlying mechanism, BM-MSCs were treated with DAPT at $0,2.5,5$ and $10 \mu \mathrm{M}$ for 72 $\mathrm{h}$, we found that there was a low to moderate basal level of autophagy in BM-MSCs. DAPT treatment of BM-MSCs induced remarkable autophagy in a concentration-dependent manner.

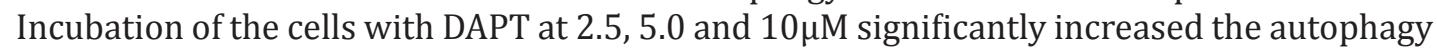
related proteins LC3II/I and Beclin-1 respectively, compared to the control cells. Meanwhile, we also investigated the effect of DAPT treatment on PI3K/Akt/mTOR pathway expression in BM-MSCs. With increasing concentrations of DAPT, The p-PI3K/PI3K ratio was significantly decreased, indicating a clear dose dependence of PI3K decrease by DAPT. As a downstream 


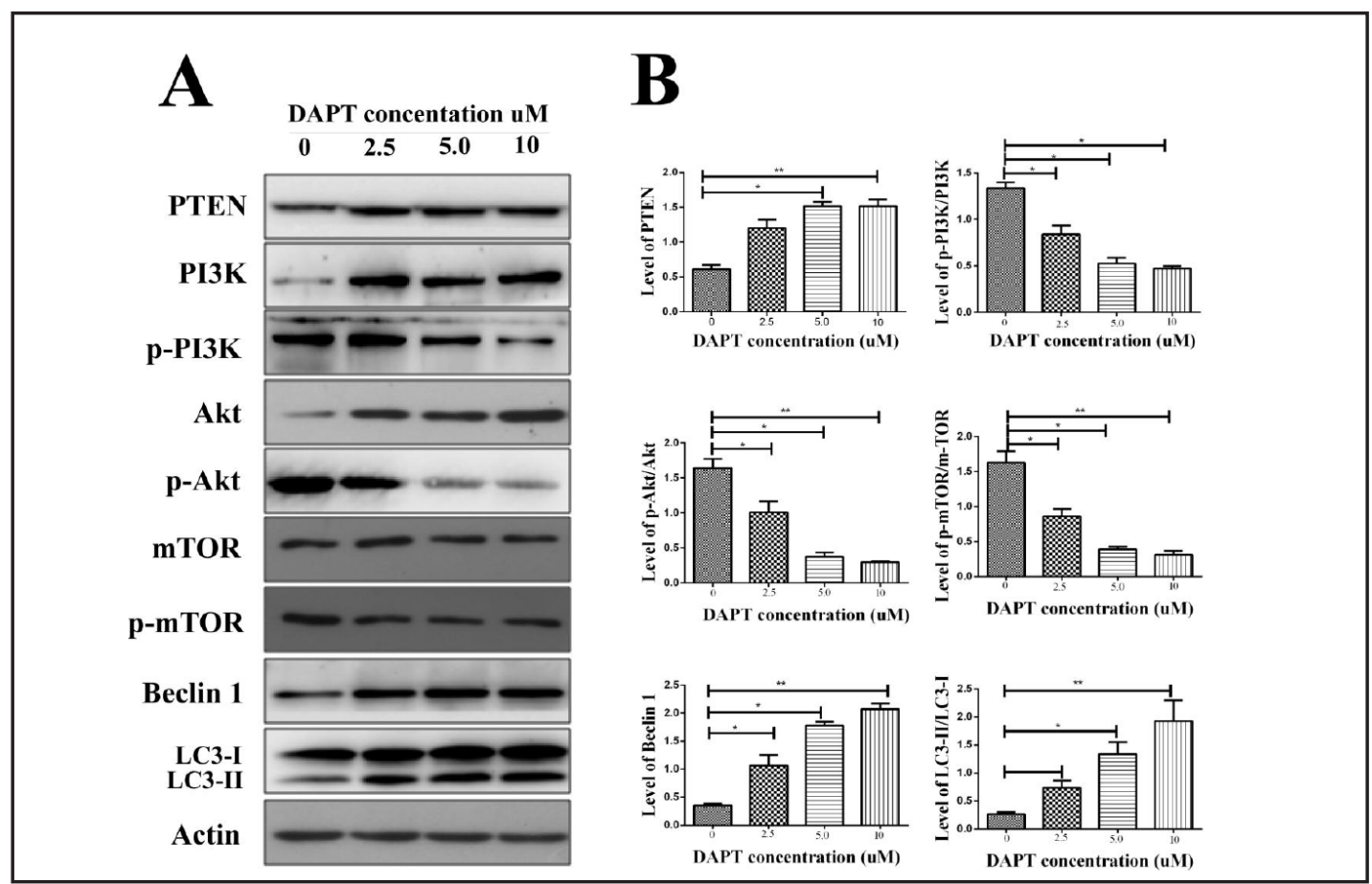

Fig. 5. Effect of DAPT treatment on the expression levels of PTEN, phosphorylated PI3K, PI3K, phosphorylated Akt, Akt, phosphorylated mTOR , mTOR, beclin-1 and LC3-I, LC3-II in BM-MSCs. (A) Representative blots of the proteins measured. (B) Levels of the proteins PTEN, Beclin 1 and ratios of phosphorylated PI3K, phosphorylated Akt, and phosphorylated mTOR over the corresponding protein without phosphorylation and LC3-II over LC3-I, $\beta$-actin was used as the internal control. ${ }^{*} \mathrm{p}<0.05,{ }^{* *} \mathrm{p}<0.01$.

effector of PI3K, Akt can activate mTOR, DAPT at 2.5-10 $\mu \mathrm{M}$ did not significantly affect the expression of Akt. However a reducing phosphorylation of Akt was observed, indicating a clear dose dependence of Akt phosphorylation inhibition by DAPT. The expression of PTEN, a negative regulator of the PI3K/Akt pathway, in BM-MSCs was significantly increased by DAPT at $0.5,2.5$, and $10 \mu \mathrm{M}$. mTOR plays a key role in cell growth, autophagic cell death, and homeostasis, incubation of BM-MSCs with DAPT at 2.5-10 $\mu \mathrm{M}$, the ratio of phosphorylation of mTOR level was significantly decreased in comparison with the control cells (Fig. 5A, B).

Chloroquine and 3-methyladenine represse DAPT-induced autophagy

To identify that increased autophagosome formation by DAPT treatment was due to increased autophagic flux, BM-MSCs were treated with autophagy inhibitor CQ and 3-MA. CQ inhibits the last step in autophage induced additional accumulation of LC3-II, meanwhile 3-MA blocks the formation of autophagosomes by inhibiting the class III phosphatidylinositol 3-kinase. The addition of CQ accumulated LC3-II expression and 3-MA significantly reduced the expression of LC3-II.

As expected, compared with the control group or DMSO-alone group, autophage activity was increased in the DAPT group with a high LC3-II/LC3-I ration and beclin-1 expression. The addition of CQ accumulated LC3-II expression in BM-MSCs pretreated with DAPT and 3-MA significantly reduced the expression of LC3-II (Fig. 6A, B). The DAPT-induced abnormal expression of Beclin1 was significantly reduced by CQ and 3-MA (Fig. 6A, B). We also measured the autophagic vacuole formation by immunofluorescent staining. The results showed that addition of DAPT drastically increased the accumulation of LC3-II expression and the formation of LC3 puncta, compared to the control group. Treatment of the cells with CQ and 3-MA significantly reduced the DAPT-induced autophagy (Fig. 6C), suggesting that DAPT induced autophagy can be repressed significantly by CQ and 3-MA. 


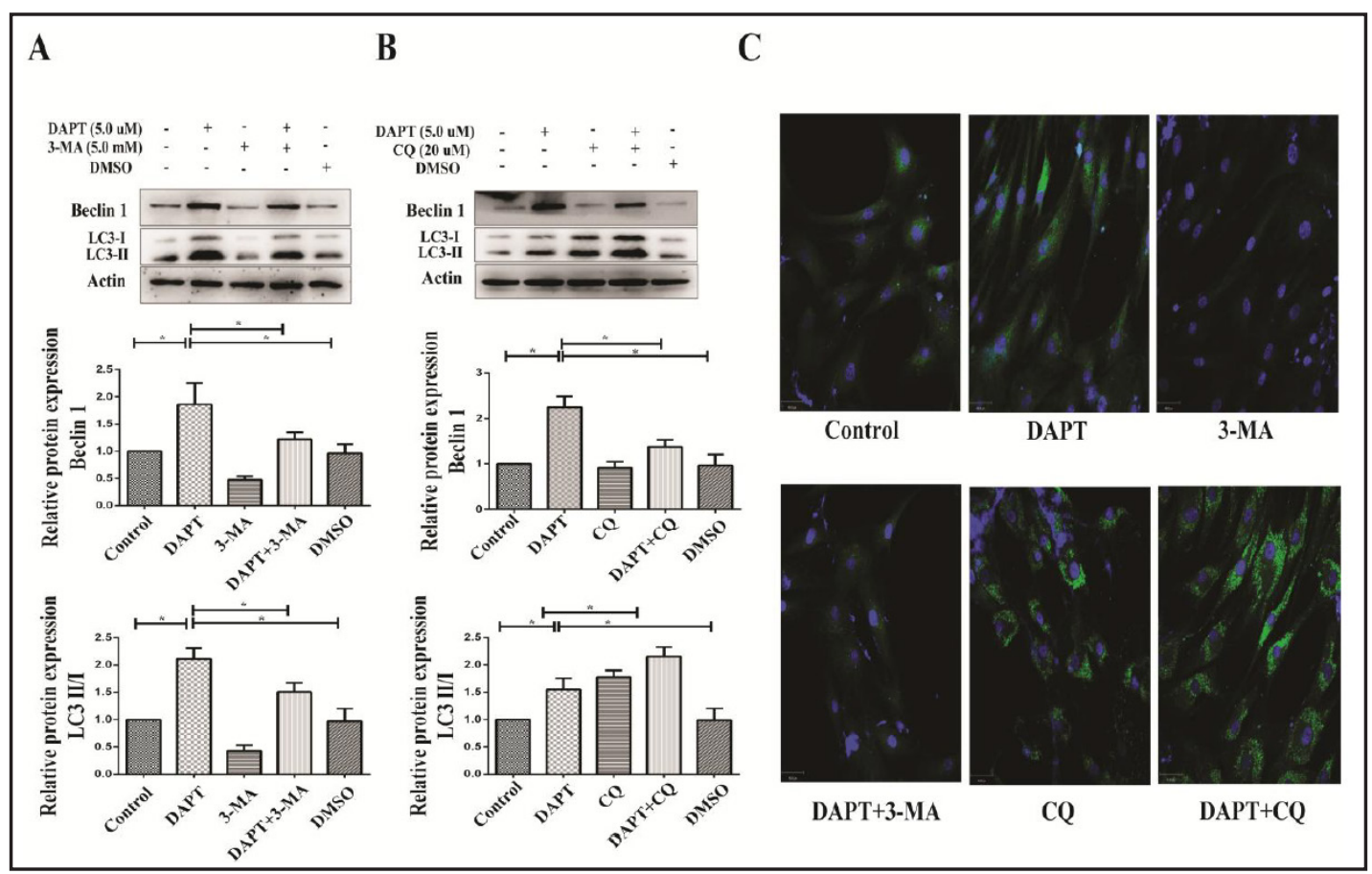

Fig. 6. Cells were treated with DAPT $(5 \mu \mathrm{M})$ alone or combination with 3-MA (5mM) and CQ $(20 \mu \mathrm{M})$ for 72 h. A. Western blot showing the effect of CQ and 3-MA on Beclin-1 and LC3II/I protein levels in DAPT treated BM-MSCs; B. LC3 protein levels were analyzed by confocal microscopy, Original magnification, $600 \times .{ }^{*} \mathrm{p}$ $<0.05,{ }^{* *} \mathrm{p}<0.01$.

\section{Discussion}

Adipogenesis, the formation of adipose tissue, has an important impact on different biological aspects of aging, insulin sensitivity, lipid metabolism, stress response and inflammation [27]. The imbalance of adipogenesis is often associated with pathophysiological conditions, such as obesity, aging, aplastic anemia and hematological malignancies [28, 29]. The regulatory mechanisms of adipogenic differentiation of MSCs remain to be elucidated. The present study demonstrated that inhibition of Notch signaling promoted the adipogenic differentiation of BM-MSCs. In addition, the autophagy plays an important role in DAPTinduced adipogenic differentiation of BM-MSCs involving PTEN-PI3K/Akt/mTOR pathway.

The Notch signaling is highly conserved among multi-cellular organisms and has been demonstrated to participate in a broad range of developmental processes in part through the regulation of cell fate decisions [30]. To clarify the molecular mechanism of how Notch signaling mediates adipocytes differentiation, we focused on the well-known signaling pathways of the phosphatidylinositol 3-OH kinase (PI3K) and the serinehreonine kinase AKT, which are key intermediates of several cell-extrinsic signals that are involved in cell growth and survival [24]. Several lines of evidence now point to a link between the Notch and PI3K pathways via PTEN, a negative regulator of the PI3K pathway [31, 32]. Of interest several reports indicated that the Notch and PI3K signaling pathways interact at several levels and in various cellular contexts $[33,34]$. One of Akt's key targets is the mTOR pathway. mTOR belongs to a family of PI3K-related kinases and is a key regulator of cell growth and metabolism. It resides in two distinct signaling complexes, TORC1 and TORC2 $[35,36]$. The inhibitory function of mTOR complex 1 (mTORC1) in autophagy is well established. Therefore, mTORC1 regulates autophagy is of great importance because it may link others signals to regulation of autophagy [37]. Autophagy is essential under both physiological and pathological conditions. Basal autophagy exerts "quality control" function, that is, eliminating old organelles or turning over long-lived proteins, to maintain 


\section{Cellular Physiology Cell Physiol Biochem 2015;36:1991-2002 \\ \begin{tabular}{ll|l} 
and Biochemistry & $\begin{array}{l}\text { DOI: 10.1159/000430167 } \\
\text { Publisned onIIne: July 17, } 2015\end{array}$ & $\begin{array}{l}\text { C 2015 S. Karger AG, Basel } \\
\text { www.karger.com/cpb }\end{array}$ \\
\cline { 2 - 3 } Song et al.: Notch Signaling Regulates Adipogenesis via Autophagy and PTEN-PI3K/
\end{tabular} AKT/mTOR Pathway}

cellular homeostasis. Autophagy has also been involved in adipogenic differentiation, since its impairment in Atg5 and Atg7 knockout mice compromises fat cell maturation [38, 39]. Furthermore, autophagy also favors adipogenic differentiation by affecting cellular shape [40]. The relationship between autophagy and adipogenesis has been characterized in various contexts, however, it has not been extensively studied in MSCs [15, 16, 41]. Therefore autophagy may involve the modulation of the Notch signaling pathway in the adipogenic differentiation of mesenchymal stem cells.

In the first part of this study, we investigated Notch signaling and autophagy alterations during adipocyte differentiation. The reduced gene expression of Notch receptors NOTCH1, NOTCH2, NOTCH3, Notch canonical ligands DLL1, DLL3, DLL4, the two non-canonical ligands DLK1, DLK2, and downstream effector HEY1 observed during adipocyte differentiation. Meanwhile, basal autophagy increased during the initial stages of adipogenic differentiation of BM-MSCs. Next, we found that when BM-MSCs were treated with Notch inhibitor DAPT for 14 days in the presence of adipogenic induction media, there was a significant increase in adipocytes as evidenced by increase in Oil Red $O$ staining positive cells as well as a higher mRNA levels for PPAR $\gamma$ and C/EBP $\alpha$. Meanwhile, DAPT treatment of BM-MSCs induced a remarkable autophagy in a concentration-dependent manner via inhibition of PI3K/Akt/ mTOR pathway. Based on our data, we hypothesized that Notch inhibitor DAPT-mediated promotion of adipogenesis might be caused by the early autophagy activation. In order to determine whether the DAPT-induced autophagy could affect the differentiation potential of BM-MSCs, we treated BM-MSCs with autophagy inhibitor CQ and 3-MA for early 3 days and allowed the cells to differentiate into adipocytes in the addition of DAPT. The results showed that CQ and 3-MA could inhibit DAPT-induced autophagy, and significantly undermine the effects of DAPT-induced adipogenic differentiation of BM-MSCs. Therefore, our data showed that Notch signaling plays an important role in BM-MSCs adipogenic differentiation via early autophagy activation partly involves PTEN-PI3K-Akt-mTOR signaling pathway.

One limitation of this study is the lack of Notch receptor and ligand protein expression data, and didn't use specific inhibitors of such downstream proteins to block DAPT effect, so the actual regulatory effects controlling MSC adipogenesis of Notch signaling could not be determined either. Further studies are needed to explore the role of Notch signaling in regulating the adipogenic differentiation of MSCs.

Although previous studies have demonstrated that inhibition of Notch altered differentiation of MSCs and emphasized the important role for autophagy in the differentiation of MSCs. The current study not only confirmed the importance of Notch signaling in promoting MSCs adipogenesis, but also provides detailed evidence for autophagy involving the modulation of the Notch signaling pathway in the adipogenic differentiation of MSCs through the PTEN-PI3K/Akt/mTOR pathway. These results bring new insights into our understanding of the Notch signaling regulation of BM-MSCs adipogenesis appearing spontaneously during aging or pathological conditions, and suggest a clinical potential of MSCs in regenerative medicine.

\section{Disclosure Statement}

No potential conflicts of interest were disclosed.

\section{Acknowledgments}

This work was supported by the National Natural Science Foundation of China (81330015 and 31470951) and The National Basic Research Program of China (973 Program) (2011CB964802) and National Key Technology Research and Development Program (Grant No. 2013BAI01B09). 


\section{Cellular Physiology Cell Physiol Biochem 2015;36:1991-2002 \begin{tabular}{l|l} 
and Biochemistry Published onlIne: July 17, 2015 & $\begin{array}{l}\text { C) 2015 S. Karger AG, Basel } \\
\text { www.karger.com/cpb }\end{array}$ \\
\hline
\end{tabular}}

Song et al.: Notch Signaling Regulates Adipogenesis via Autophagy and PTEN-PI3K/ AKT/mTOR Pathway

\section{References}

1 Bianco P, Robey PG, Simmons PJ: Mesenchymal stem cells: Revisiting history, concepts, and assays. Cell stem Cell 2008;2:313-319.

-2 Levi B, Longaker MT: Concise review: Adipose-derived stromal cells for skeletal regenerative medicine. Stem Cells 2011;29:576-582.

- 3 Ye L, Fan Z, Yu B, Chang J, Al Hezaimi K, Zhou X, Park NH, Wang CY: Histone demethylases KDM4B and KDM6B promotes osteogenic differentiation of human MSCs. Cell stem Cell 2012;11:50-61.

4 Li J, Yang S, Lu S, Zhao H, Feng J, Li W, Ma F, Ren Q Liu B, Zhang L, Zheng Y, Han ZC: Differential gene expression profile associated with the abnormality of bone marrow mesenchymal stem cells in aplastic anemia. PloS One 2012;7:e47764.

-5 Liao L, Yang X, Su X, Hu C, Zhu X, Yang N, Chen X, Shi S, Shi S, Jin Y: Redundant miR-3077-5p and miR705 mediate the shift of mesenchymal stem cell lineage commitment to adipocyte in osteoporosis bone marrow. Cell Death Dis 2013;4:e600.

6 Huang JS, Mulkern RV, Grinspoon S: Reduced intravertebral bone marrow fat in HIV-infected men. AIDS 2002;16:1265-1269.

7 Rippo MR, Babini L, Prattichizzo F, Graciotti L, Fulgenzi G, Tomassoni Ardori F, Olivieri F, Borghetti G, Cinti S, Poloni A, Fazioli F, Procopio AD: Low Fasl levels promote proliferation of human bone marrowderived mesenchymal stem cells, higher levels inhibit their differentiation into adipocytes. Cell Death Dis 2013;4:e594.

8 Kluge R, Scherneck S, Schurmann A, Joost HG: Pathophysiology and genetics of obesity and diabetes in the New Zealand obese mouse: A model of the human metabolic syndrome. Methods Mol Biol 2012;933:59-73. Chou WW, Wang YT, Liao YC, Chuang SC, Wang SN, Juo SH: Decreased microRNA-221 is associated with high levels of TNF- $\alpha$ in human adipose tissue-derived mesenchymal stem cells from obese woman. Cell Physiol Biochem 2013;32:127-137.

-10 Chen Q, Shou P, Zhang L, Xu C, Zheng C, Han Y, Li W, Huang Y, Zhang X, Shao C, Roberts AI, Rabson AB, Ren G, Zhang Y, Wang Y, Denhardt DT, Shi Y: An osteopontin-integrin interaction plays a critical role in directing adipogenesis and osteogenesis by mesenchymal stem cells. Stem Cells 2014;32:327-337.

-11 Chen Y, Gao H, Yin Q, Chen L, Dong P, Zhang X, Kang J: ER stress activating ATF4/CHOP-TNF- $\alpha$ signaling pathway contributes to alcohol-induced disruption of osteogenic lineage of multipotential mesenchymal stem cell. Cell Physiol Biochem 2013;32:743-754.

12 Wang J, Guan X, Guo F, Zhou J, Chang A, Sun B, Cai Y, Ma Z, Dai C, Li X, Wang B: miR-30e reciprocally regulates the differentiation of adipocytes and osteoblasts by directly targeting low-density lipoprotein receptor-related protein 6. Cell Death Dis 2013;4:e845.

13 Klionsky DJ, Abdalla FC, Abeliovich H, Abraham RT, Acevedo-Arozena A, Adeli K, Agholme, et al.: Guidelines for the use and interpretation of assays for monitoring autophagy. Autophagy 2012;8:445-544.

-14 Arsov I, Adebayo A, Kucerova-Levisohn M, Haye J, MacNeil M, Papavasiliou FN, Yue Z, Ortiz BD: A role for autophagic protein beclin 1 early in lymphocyte development. J Immunol 2011;186:2201-2209.

15 Zhang Y, Zeng X, Jin S: Autophagy in adipose tissue biology. Pharmacol Res 2012;66:505-512.

-16 Zhang C, He Y, Okutsu M, Ong LC, Jin Y, Zheng L, Chow P, Yu S, Zhang M, Yan Z: Autophagy is involved in adipogenic differentiation by repressesing proteasome-dependent PPAR $\gamma 2$ degradation. Am J Physiol Endocrinol Metab 2013;305:E530-539.

17 Fiuza UM, Arias AM: Cell and molecular biology of Notch. J Endocrinol 2007;194:459-474.

- 18 Park JS, Kim SH, Kim K, Jin CH, Choi KY, Jang J, Choi Y, Gwon AR, Baik SH, Yun UJ, Chae SY, Lee S, Kang YM, Lee KC, Arumugam TV, Mattson MP, Park JH, Jo DG: Inhibition of notch signalling ameliorates experimental inflammatory arthritis. Ann Rheum Dis 2013;74:267-274

19 Fortini ME: Notch signaling: The core pathway and its posttranslational regulation. Dev Cell 2009;16:633647.

20 Ding XY, Ding J, Wu K, Wen W, Liu C, Yan HX, Chen C, Wang S, Tang H, Gao CK, Guo LN, Cao D, Li Z, Feng GS, Wang HY, Xu ZF: Cross-talk between endothelial cells and tumor via delta-like ligand 4/Notch/PTEN signaling inhibits lung cancer growth. Oncogene 2012;31:2899-2906.

-21 Kunnimalaiyaan M, Chen H: Tumor suppressor role of Notch-1 signaling in neuroendocrine tumors. Oncologist 2007;12:535-542. 


\section{Cellular Physiology Cell Physiol Biochem 2015;36:1991-2002 \begin{tabular}{l|l} 
and BOI: 10.1159/000430167 & $\begin{array}{l}\text { C } 2015 \text { S. Karger AG, Basel } \\
\text { www.karger.com/cpb }\end{array}$ \\
\hline
\end{tabular}}

Song et al.: Notch Signaling Regulates Adipogenesis via Autophagy and PTEN-PI3K/ AKT/mTOR Pathway

22 Jiao Z, Wang W, Hua S, Liu M, Wang H, Wang X, Chen Y, Xu H, Lu L: Blockade of notch signaling ameliorates murine collagen-induced arthritis via suppressing Th1 and Th17 cell responses. Am J Pathol 2014;184:1085-1093

23 Medyouf H, Gao X, Armstrong F, Gusscott S, Liu Q, Gedman AL, Matherly LH, Schultz KR, Pflumio F, You MJ, Weng AP: Acute T-cell leukemias remain dependent on Notch signaling despite PTEN andINK4A/ARF loss. Blood 2010;115:1175-1184.

24 Cornejo MG, Mabialah V, Sykes SM, Khandan T, Lo Celso C, Lopez CK, Rivera-Munoz P, Rameau P, Tothova Z, Aster JC, DePinho RA, Scadden DT, Gilliland DG, Mercher T: Crosstalk between Notch and AKT signaling during murine megakaryocyte lineage specification. Blood 2011;118:1264-1273.

-25 Roderick JE, Gonzalez-Perez G, Kuksin CA, Dongre A, Roberts ER, Srinivasan J, Andrzejewski C, Jr., Fauq AH, Golde TE, Miele L, Minter LM: Therapeutic targeting of NOTCH signaling ameliorates immune-mediated bone marrow failure of aplastic anemia. J Exp Med 2013;210:1311-1329.

-26 Duan L, Ren Y: Role of notch signaling in osteoimmunology--from the standpoint of osteoclast differentiation. Eur J Orthod 2013;35:175-182.

-27 Ullah M, Stich S, Haupl T, Eucker J, Sittinger M, Ringe J: Reverse differentiation as a gene filtering tool in genome expression profiling of adipogenesis for fat marker gene selection and their analysis. PloS One 2013;8:e69754.

28 Battula VL, Chen Y, Cabreira Mda G, Ruvolo V, Wang Z, Ma W, Konoplev S, Shpall E, Lyons K, Strunk D, Bueso-Ramos C, Davis RE, Konopleva M, Andreeff M: Connective tissue growth factor regulates adipocyte differentiation of mesenchymal stromal cells and facilitates leukemia bone marrow engraftment. Blood 2013;122:357-366.

-29 Kordes C, Sawitza I, Gotze S, Haussinger D: Hepatic stellate cells support hematopoiesis and are liverresident mesenchymal stem cells. Cell Physiol Biochem 2013;31:290-304.

-30 Wilson A, Radtke F: Multiple functions of Notch signaling in self-renewing organs and cancer. FEBS Lett 2006;580:2860-2868.

-31 Trotman LC, Alimonti A, Scaglioni PP, Koutcher JA, Cordon-Cardo C, Pandolfi PP: Identification of a tumour suppressor network opposing nuclear Akt function. Nature 2006;441:523-527.

-32 Rossi DJ, Weissman IL: Pten, tumorigenesis, and stem cell self-renewal. Cell 2006;125:229-231.

33 Bailis W, Pear WS: Notch and PI3K: How is the road traveled? Blood 2012;120:1349-1350.

- 34 Lee K, Nam KT, Cho SH, Gudapati P, Hwang Y, Park DS, Potter R, Chen J, Volanakis E, Boothby M: Vital roles of mTOR complex 2 in notch-driven thymocyte differentiation and leukemia. J Exp Med 2012;209:713-728.

- 35 Zoncu R, Efeyan A, Sabatini DM: mTOR: From growth signal integration to cancer, diabetes and ageing. Nat Rev Mol Cell Biol 2011;12:21-35.

-36 Shepherd C, Banerjee L, Cheung CW, Mansour MR, Jenkinson S, Gale RE, Khwaja A: PI3K/mTOR inhibition upregulates NOTCH-MYC signalling leading to an impaired cytotoxic response. Leukemia 2013;27:650-660.

- 37 Alers S, Loffler AS, Wesselborg S, Stork B: Role of AMPK-mTOR-Ulk1/2 in the regulation of autophagy: Cross talk, shortcuts, and feedbacks. Mol Cell Biol 2012;32:2-11.

- 38 Baerga R, Zhang Y, Chen PH, Goldman S, Jin S: Targeted deletion of autophagy-related 5 (atg5) impairs adipogenesis in a cellular model and in mice. Autophagy 2009;5:1118-1130.

-39 Zhang Y, Goldman S, Baerga R, Zhao Y, Komatsu M, Jin S: Adipose-specific deletion of autophagy-related gene 7 (atg7) in mice reveals a role in adipogenesis. Proc Natl Acad Sci U S A. 2009;106:19860-19865.

40 Singh R, Xiang Y, Wang Y, Baikati K, Cuervo AM, Luu YK, Tang Y, Pessin JE, Schwartz GJ, Czaja MJ: Autophagy regulates adipose mass and differentiation in mice. J Clin Invest 2009;119:3329-3339.

41 Oliver L, Hue E, Priault M, Vallette FM: Basal autophagy decreased during the differentiation of human adult mesenchymal stem cells. Stem Cells Dev 2012;21:2779-2788. 